Int. J. Dev. Biol. 62: 369-372 (2018)

https://doi.org/10.1387/ijdb.180031mm

\title{
The evolution of regeneration - where does that leave mammals?
}

\author{
MALCOLM MADEN* \\ Department of Biology \& UF Genetics Institute, University of Florida, USA
}

\begin{abstract}
This brief review considers the question of why some animals can regenerate and others cannot and elaborates the opposing views that have been expressed in the past on this topic, namely that regeneration is adaptive and has been gained or that it is a fundamental property of all organisms and has been lost. There is little empirical evidence to support either view, but some of the best comes from recent phylogenetic analyses of regenerative ability in Planarians which reveals that this property has been lost and gained several times in this group. In addition, a nonregenerating species has been induced to regenerate by altering only one signaling pathway. Extrapolating this to mammals it may be the case that there is more regenerative ability in mammals than has typically been thought to exist and that inducing regeneration in humans may not be as impossible as it may seem. The regenerative abilities of mammals is described and it turns out that there are several examples of classical epimorphic regeneration involving a blastema as exemplified by the regenerating Urodele limb that can be seen in mammals. Even the heart can regenerate in mammals which has long been considered to be a property unique to Urodeles and fish and several recent examples of regeneration have come from recent studies of the spiny mouse, Acomys, which are discussed here. It is suggested that a much more thorough phylogenetic analysis of mammalian regeneration would likely reveal some important insights into the evolution of regeneration.
\end{abstract}

KEY WORDS: evolution, regeneration, mammal, Planarian, spiny mouse

One of the most enduring questions in the regeneration field must surely be: why can some organisms regenerate and others cannot? This question has been debated for more than 100 years and although there is little empirical evidence to support one view or the other this does not detract from the fascination with the question as well as its importance for regenerative medicine.

One view is that regeneration is a fundamental property of all organisms and it has been lost during evolution in many species as a result of selection against because it may have been detrimental or simply neutral with regard to survival and so it would have been eliminated. This is the popular contemporary view (Bely \& Nyberg, 2010) and stretches back to the views of Morgan (1901, as discussed in Goss, 1991, 1992). Bely \& Nyberg have concluded that the metazoan ancestor, the bilaterian ancestor, the deuterostome ancestor and the lophotrochozoan ancestor were all capable of extensive regeneration. It has been lost in the ecdysozoa and in the chordates is present in some urochordates and amphibians but has been lost in birds and mammals. If this is indeed the case, then examples of regeneration should all resemble each other and to a great degree, at least in vertebrates, they do. Regeneration of complex tissues such as limbs and tails proceeds by rapid epi- dermal wound healing, dedifferentiation of mesodermal cells and release of stem cells to generate a blastema, proliferation of the blastema, induction of signaling pathways to pattern the blastema and redifferentiation of tissues in a proximal to distal sequence. There is also a neurotrophic requirement which is considered to be the provision of a proliferation-inducing neurotrophic molecule from the nerves or Schwann cells (Farkas et al., 2016). But what about organs that do not produce a blastema during regeneration, for example the skin which regenerates after epithelial wound closure over a scab (if present) but the dermal fibroblasts do not seem to accumulate together to form a blastema even though they proliferate extensively during skin regeneration?. Likewise the replacement of cardiomyocytes in the heart requires a stimulus from the epicardium but there is no blastema-like structure and again with lens regeneration where only one cell type is involved and here the process only involves dedifferentiation, proliferation and redifferentiation. So a blastema may not be an absolute requirement for epimorphic regeneration as long as proliferation can

Abbreviations used in this paper: ac, apical cap; bl, blastema.

*Address correspondence to: Malcolm Maden. Department of Biology \& UF Genetics Institute, University of Florida, USA. e-mail: malcmaden @ ufl.edu

Submitted: 12 January, 2018; Accepted: 16 January, 2018.

ISSN: Online 1696-3547, Print 0214-6282

(c) 2018 UPV/EHU Press

Printed in Spain 
occur in an orderly fashion. Nevertheless, this view of regeneration is a very positive one from the point of view of the ultimate aim of inducing regeneration in humans because if all regenerative processes are fundamentally the same then studies on the axolotl are highly relevant to inducing the property in humans. It also means that in non-regenerating organisms such as humans the ability to regenerate has likely been blocked and to permit regeneration we 'only' have to find out how to unblock the process rather than re-invent the wheel.

The alternative view, that evolution has selected for each example of regeneration independently and that it arose separately as an adaptation to environmental pressures has also been a popular view over time. This adaptive property of regeneration means that in animals that frequently lose parts such as appendages the ability to regenerate them is a selective advantage and this concept is frequently discussed in terms of the more a structure is liable to be injured in nature the more likely it is to be able to regenerate. This certainly applies to the lizard tail which is apparently frequently lost in nature - as frequently as $85 \%$ in some estimates (Vitt \& Cooper, 1986) and many marine invertebrates in which a large proportion of the population (97\%) may be regenerating at any one time (Clark et al., 2007). T.H. Morgan was not an advocate of this view and set out to test this in hermit crabs which only expose the first two pairs of legs while the remaining three pairs are protected inside the shell (discussed in Goss, 1992). Yet the third, fourth and fifth leg can regenerate as well as the first two. Similarly, now we know that internal organs of lower vertebrates can regenerate such as the heart, the lens or the forebrain this begs the question: how many times had the heart been damaged in order for regeneration to be adaptive in this circumstance or the lens and so on? This adaptive hypothesis has very different implications from the innate hypothesis above for the practicalities of inducing regeneration in humans because if each system has evolved separately then they are unique in their mechanisms and learning about axolotl limb regeneration may have no relevance to anything else or learning how to induce regeneration in mice as a model system may have no relevance to inducing it in humans.

Some of the most relevant studies in relation to these two arguments have been conducted on Planarians. The regenerative abilities of Planarians are legendary and were the subject of T.H. Morgan's studies on regeneration. However, regenerative ability is not universal amongst Planarians as many species are incapable of regenerating and a phylogenetic analysis of 60 regenerating and non-regenerating species by J. Rink (pers.comm.) has revealed this patchy distribution. There are clear examples where some branches of the tree can no longer regenerate and the ability must have been lost and there are also examples of where subsequent branches of the tree can regenerate and the ability must have been regained. This analyses of large numbers of closely related species reveals that it may not be so hard to gain or lose regenerative ability as we might imagine as this has happened several times in Planarians. Indeed the molecular basis of this property has been determined for one species which cannot regenerate its head, Dendrocoelum lacteum (Liu et al., 2013). After amputation of the head the initial phases of regeneration, wound healing and establishment of a blastema occurred normally but what goes wrong is the subsequent lack of expression of head fate specification genes and the continued expression of tail specification genes. Since the inhibition of Wnt/ $\beta$-catenin is required for head specification in other regenerating Planarians the inhibition of $\beta$-catenin signaling was all that was required to turn this non-regenerating species into a regenerating one. A remarkably simple requirement for one gene on the Wnt pathway in amongst all the thousands of genes required for regeneration. This experiment shows that it may not be so hard to gain regenerative ability as we might imagine and gives great impetus to the search for ways of inducing regeneration in mammals. It also suggests that the most likely scenario for the evolution of regeneration - innate or adaptive - is the former.

This type of phylogenetic analysis really needs to be done in mammals to see if these same characteristics apply. The common view is that mammals (and birds) do not regenerate, but that depends on what you mean by regeneration. At the molecular or cellular level, regeneration is ubiquitous to multicellular organisms - molecular turnover of membranes and proteins, turnover of cell types such as blood cells, continual replacement of the surface epithelium or the gut lining, slow turnover of resident stem cell populations, and universal ability of epithelia to heal over wounds. This is physiological regeneration. At first sight what seems to have been lost is the ability to regrow organs or complex tissues. But is this true or have regenerative abilities never been tested in mammals because it is assumed that they do not?

There are already some striking examples of anatomically complex tissues that can regenerate in mammals. The annual replacement of deer antlers is surely one of the most conspicuous examples and antler regeneration shows many features typical of regeneration in lower vertebrates (Goss, 1983). After shedding the antler the wound is healed by migration of adjacent skin over the pedicle which does not form a scar but instead is an active and inductive tissue. Dermal cells and periosteal cells intermingle in the healing area which will grow into the new antler bud and there are extensive down-pushings of the epithelium at the tip into the mesoderm of the bud reminiscent of the apical cap of the limb blastema. If the wound skin is damaged it can give rise to a small ectopic antler and if normal full-thickness skin is grafted over the pedicle stump then antler regeneration is inhibited. The pedicle can be transplanted to other regions of the body where it will form an ectopic antler. New antler growth is derived from fibroblast-like cells derived from the periosteum of the pedicle (maybe also from the dermis of the healing skin) and the cells at the tip resemble blastemal cells in their undifferentiated nature and high proliferation rate. The rapidly growing antler is covered in velvet, (regenerated non-scarring skin) which contains glands and hairs (minus erector pili muscles) that are formed de novo at the tip of the growing antler. Therefore this skin is a highly regenerative tissue. Thus antlers show many of the features of lower vertebrate regeneration and could be an amazing system for studying mammalian regeneration were it not for their obvious drawbacks as a model organism.

The generation of a blastema is the hallmark of complex tissue regeneration but this is not the only regenerating mammalian system with a blastema because the digit tips of mice (Borgens, 1982; Neufeld \& Zhou 1995; Muneoka et al., 2008), rats (Said et al., 2004), monkeys (Singer etal., 1987) and humans (Illingworth, 1974; Rinkevich et al., 2015) can regenerate by a process which seems remarkably similar to that of the antler. After re-epithelialization, a blastema is formed consisting mostly of osteoblasts formed by bone breakdown which then proliferates and redifferentiates into new bone. The blastemal cells are lineage restricted rather than dedifferentiating to a multipotent/totipotent cell type (Lehoczky et 
al., 2011; Rinkevitch et al., 2011) and proliferate under the influence of Wnt signaling from the nail bed (Takeo et al., 2013) and factors secreted by nerve-associated Schwann cells (Johnston etal., 2016). Thus the presence of a blastema in antler and digit regeneration as well as the regenerating limb of Urodeles suggests that similar processes may be going on both in mammals and axolotls.

We have recently described another blastema that appears in mammals, during regeneration of ear punches of spiny mice, Acomys (Fig. $1 \mathrm{~A}, \mathrm{~B}$ ). A complex arrays of tissues is regenerated from this blastema - cartilage, skeletal muscle, dermis, hairs and sebaceous glands (Seifert et al., 2012). This phenomenon was first observed in rabbits (Voronstova \& Liosner, 1960; Joseph \& Dyson 1966; Goss \& Grimes, 1972) and subsequently has been observed in all species of Acomys examined (Gawriluk et al., 2016), athymic nude mice (Gawronska-Kozak, 2004) in small holes of the MRL mouse ear (Clarke et al., 1998) and in chinchillas, cows, pigs (Williams-Boyce \& Daniel, 1986), pikas and bats (Goss, 1987). Even more remarkably like the amphibian limb blastema this ear blastema shows neurotrophic dependence because if the auricular nerve is

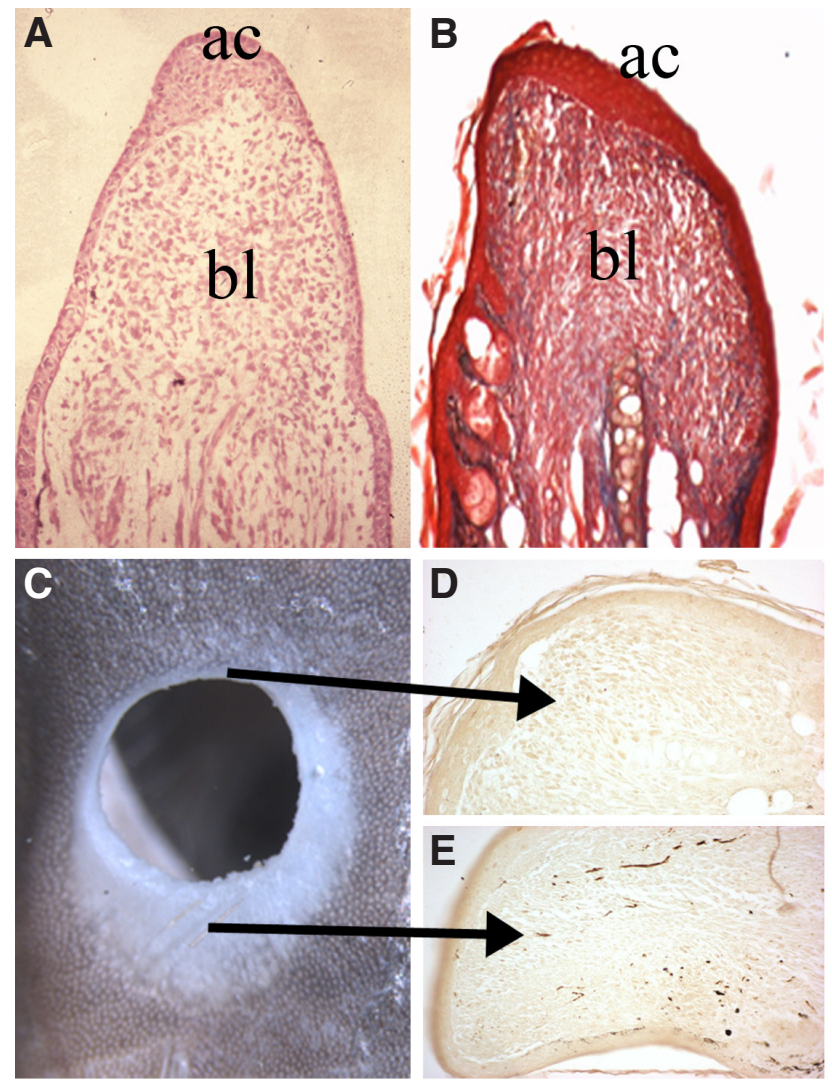

Fig. 1. Axolotl and mammalian blastemas. (A) A blastema (b/) growing on the amputated limb of a juvenile axolotl. Ac, apical cap. (B) A blastema (bl) growing on the regenerating ear of Acomys. Ac, thickened apical epidermis which resembles the apical cap of the regenerating limb in (A). (C) The regenerating ear punch in Acomys showing eccentric growth with almost all of the regeneration coming from the proximal semi-circle llower arrow) and virtually none from the distal segment of the circle (upper arrow). (D) Section through the distal segment of the regenerating ear stained with RT97 antibody to identify nerve fibres. None are present here. (E) Section through the distal segment of the regenerating ear stained with RT97 antibody to identify nerve fibres. There are many fibres present here. cut then the ear punch does not regenerate (Buckley et al., 2011). This phenomenon can also be seen during normal regeneration of the ear punch in Acomys. After the damage the proximal half of the circle (closest to the head) regenerates quite fast and the distal half of the circle (farthest from the head) hardly regenerates at all (Fig. 1C) and the hole finally closes in a very distal position. Sectioning these segments of the regenerating ear and staining for nerve fibres reveals that the proximal blastema which does all of the regenerating is riddled with nerve fibres (Fig. 1E) whereas they are completely absent in the distal blastema which hardly grows at all (Fig. 1D) (Gawriluk et al., 2016).

If the skin regrows perfectly over the ear punches in these animals then the body skin ought to regenerate as well. This has been reported in rabbits (Billingham \& Russell, 1956) without a histological study, but has been well documented in Acomysspecies (Seifert et al., 2012; Brant et al., 2015; 2016). Following various sizes of full thickness wounds as well as full thickness burns in Acomys the wound epithelium covers the wound twice as fast as the rate of Mus wounds and within two weeks new hair follicles can be seen developing across the wound which are undergoing Wnt7a signaling. These newly regenerated hair follicles deepen into the new dermis of the wound bed and produce sebaceous glands and erector pili muscles so they are fully functional. There is a much reduced inflammatory reaction in Acomys compared to Mus with far fewer cytokines and lower levels of cytokines induced, MMPs are higher in Acomys and the matrix of the wound bed is softer, expressing lower levels of collagens. Eventually even the skeletal muscle of the panniculus carnosus regenerates which is a striking behavior because the act of removing the skin creates a hole in the muscle layer which is equivalent to a volumetric muscle loss injury which does not regenerate in other mammalian laboratory organisms or humans.

Even more impressively for a mammal, heart regeneration is not just restricted to Urodeles and zebrafish but also occurs in newborn mice after either a myocardial infarction induced by ligating the coronary artery or after apex resection as in Urodeles and zebrafish (Porello et al., 2011, 2013). In mice this ability is lost at P7, but in Acomys cardiac regenerative ability is retained into adulthood. Following a myocardial infarction induced by coronary artery ligation adult Acomys show an amazing regenerative recovery. After two weeks there is a massively reduced region of scarring, the ventricular wall does not thin and the ejection fraction is completely normal. In contrast the Mus heart has a huge region of fibrosis, the ventricle wall thins excessively and there is a $71 \%$ reduction in ejection fraction (Qui et al., in preparation).

The mammalian liver also regenerates after partial hepatectomy and although this is referred to as compensatory hypertrophy because the removed parts of the liver are not regenerated instead the remaining parts expand to compensate, this is still a complex process involving the coordinated upregulation of cytokine, growth factor, and metabolic networks resembling true regeneration (Fausto et al., 2006). Similarly, the lung can undergo compensatory hypertrophy after removal of one or more of the lobes in exactly the same manner as the liver - the remaining tissue increases in alveolar number to compensate for the loss (Matsumoto et al., 2009). Interestingly this process is not a hallmark of incomplete or inefficient epimorphic regeneration as might be imagined and only seen in a supposed non-regenerating system like a mammal because the axolotl also regenerates its lung by compensatory 
hypertrophy (Monaghan, pers. comm.) even though it is the champion of blastemal based epimorphic regeneration.

There are also some other very surprising examples of mammalian organ regeneration. The mouse pancreas can regenerate after surgical removal of $60-90 \%$ of the tissue (Stocum, 2006). Skeletal muscle regenerates after a variety of injuries such as myotoxin administration or freezing, but not if complete pieces of muscle are removed, presumably because muscle regeneration requires a connective tissue framework to promote and guide regeneration, but as mentioned above Acomys can regenerate muscle after such tissue removal injuries.

It is clear therefore that there are a surprisingly large number of tissues and organs that can regenerate in mammals ranging from the heart to the skin and that is based upon a very small sample (maybe 25 species) of the 5400 species of mammals which are present on earth. What really needs to be done in mammals is a thorough phylogenetic analysis as has been done in Planarians as discussed above. It would not be surprising if many more mammals were discovered with regenerative powers and that even in mammals regenerative abilities may have been been lost and regained several times as in Planarians. If so then this is a very positive outlook for discovering how to induce regeneration in non-regenerating mammals and ultimately humans.

\section{References}

BELY A E and NYBERG K G (2009). Evolution of animal regeneration: re-emergence of a field. Trends Ecol Evol 25: 161-170.

BILLINGHAM RE and RUSSELL P S (1956). Incomplete wound contracture and the phenomenon of hair neogenesis in rabbits' skin. Nature 177: 791-792.

BORGENS R B (1982). Mice regrow the tips of their foretoes. Science 217: 747-750.

BRANT J O, LOPEZ M-C, BAKER H V, BARBAZUK W B and MADEN M (2015). A comparative analysis of gene expression profiles during skin regeneration in Mus and Acomys. PlosOne 10: e0142931.

BRANT J O, YOON J H, POLVADORE T, BARBAZUK W B and MADEN M (2016). The cellular basis of scar-free skin regeneration in the spiny mouse. Wound Rep Regen 24: 75-88.

BUCKLEY G, METCALFE A D and FERGUSON M J W (2011). Peripheral nerve regeneration in the MRL/MpJ ear wound model. J Anatomy 218: 163-172.

CLARKE L D, CLARK R K and HEBER-KATZ E (1998). A new murine model for mammalian wound repair and regeneration. Clin Immunol Immunopath88: 35-45.

CLARK M S, DUPONT S, ROSETTI H, BURNS G, THORNDYKE M C and PECK L S (2007). Delayed arm regeneration in the Antarctic brittle star Ophionotus victoriae. Aquat Biol 1: 45-53.

FARKAS J E, FREITAS P D, BRYANT D M, WHITED J L and MONAGHAN J R (2016). Neuregulin-1 signaling is essential for nerve-dependent axolotl limb regeneration. Development 143: 2724-2731.

FAUSTO N, CAMPBELL J S and RIEHLE K J (2006). Liver Regeneration. Hepatology 43: 45-53.

GAWRILUK T R, SIMKIN J, THOMPSON K L, BISWAS S K, CLARE-SALZLER Z, KIMANI J M, KIAMA S G, SMITH J J, EZENWA V O and SEIFERT A W (2016). Comparative analysis of ear-hole closure identifies epimorphic regeneration as a discrete trait in mammals. Nature Comm 7: 11164.

GOSS R J (1983). Deer Antlers: Regeneration, function and evolution. Acad Press, New York, New York.

GOSS R J (1987). Why mammals don't regenerate - or do they? NIPS 2: 112-115.

GOSS R J (1991). The natural history (and mystery) of regeneration. In 'A history of regeneration research' C.E. Dinsmore ed. Camb Univ Press 7 -23.

GOSS R J (1992). The evolution of regeneration: adaptive or inherent? J Theor Biol
159: 241-260.

GOSS R J and GRIMES L N (1972). Tissue interactions in the regeneration of rabbit ear holes. Am Zool 12: 151-157.

GOWRONSKA-KOSAK B (2004). Regeneration of the ears of immunodieficient mice: identification and lineage analysis of mesenchymal stem cells. Tissue Eng 10: 1251-1265.

ILLINGWORTH C M (1974). Trapped fingers and amputated finger tips in children. J Pediatr Surg 9: 853-858.

JOHNSTON A P, YUZWA S A, CARR M J, MAHMUD N, STORER M A, KRAUSE $M P$ et al., (2016). Dedifferentiated Schwann cell precursors secreting paracrine factors are required for regeneration of the mammalian digit tip. Cell Stem Cell 19: 433-438.

JOSEPH J and DYSON M (1966). Tissue replacement in the rabbits' ear. Br J Surg 53: 372-380.

LEHOCZKY JA, ROBERT B and TABIN C J (2011). Muse digit tip regeneration is mediated by fate-restricted progenitor cells. Proc NatIAcad SciUSA 108: 20609-20614.

LIU S-Y, SELCK C, FRIEDRICH B, LUTX R, VILA-FARRE M, DAHL A, BRANDL H, LAKSHMANAPERUMAL N, HENRY I and RINK J C (2013). Reactivating head regrowth in a regeneration-deficient planarian species. Nature 500: 81-84.

MATSUMOTO K, NAGAYASU T, HISHIKAWA Y, TAGAWA T et al.,2009). Keratinocyte growth factor accelerates compensatory growth in the remaining lung after trilobectomy in rats. J Thor Cardio Surg 137: 1499-1507.

MUNEOKAK, ALLAN C H. YANG X, LEE J and HAN M (2008). Mammalian regeneration and regenerative medicine. Birth Defects Res C Embryo Today 84: 265-280.

NEUFELD D A and ZHAO W (1995). Bone regrowth after digit tip amputation in mice is equivalent in adults and neonates. Wound Repair Regen 3: 461-466.

PORELLO E R, MAHMOUD A I, SIMPSON E, HILL J A, RICHARDSON J A, OLSONE $\mathrm{N}$ and SADEL H A (2011). Transient regenerative potential of the neonatal mouse heart. Science 331: 1078-1080.

PORELLO E R, MAHMOOD A I, SIMPSON E, JOHNSON B A, GRINSFELDER D, CANSECO D, MAMMEN P P, ROTHERMEL B A, OLSON E N and SADEL H A (2013). Regulation of neonatal and adult mammalian heart regeneration by the mir-15 family. Proc Natl Acad Sci USA 110: 187-192.

RINKEVICH Y, LINDAU P, UENO H, LONGAKER M T and WEISSMAN I L (2011). Germ-layer and lineage-restricted stem/progenitors regenerate the mouse digit tip. Nature 476: 409-413.

RINKEVICHY, MAANZN, WALMSLEY G G and SEN SK (2015). Injuries to appendage extremities and digit tips: a clinical and cellular update. Dev Dynam 244: 641-650.

SAID S, PARKE W and NEUFELD D A (2004). Vascular supplies differ in regenerating and nonregenerating amputated rodent digits. Anat. Rec. A Discov Mol Cell Evol Biol 278: 443-449.

SEIFERT A, KIAMA SG, SEIFERT M, GOHEEN J, PALMERT and MADEN M (2012). Weak skin and tissue regeneration in African spiny mice (Acomys). Nature 489: 561-566.

SHIEH S-J and CHENG T-C (2015). Regeneration and repair of human digits and limbs: fact and fiction. Regeneration 2: 149-168.

SINGER M, WECHESSER E C, GERAUDIE J, MAIER C E and SINGER J (1987). Open finger tip healing and replacement after distal amputation in rhesus monkey with comparison to limb regeneration in lower vertebrates. Anat Embryol Berl 177: 29-36.

STOCUM D L (2006). Regenerative Biology and Medicine. Academic Press.

TAKEO M, CHOU W C, SUN Q, LEE W, RABBANI P, LOOMIS C, TAKETO M M and ITO M (2013). Wnt activation in nail epithelium couples nail growth to digit regeneration. Nature 499: 228-232.

VITT L J and COOPER W E (1986). Tail loss, tail color, and predator escape in Eumeces (Lacertilia: Scincidae): age-specific differences in costs and benefits. Can J Zool 64: 583-592.

VORONSTOVA M A and LIOSNER L D (1960). Asexual propagation and regeneration. Pergamon Press, Oxford.

WILLIAMS-BOYCE P K and DANIEL J C (1986). Comparison of ear tissue regeneration in mammals. $J$ Anat 149: 55-63. 


\section{Further Related Reading, published previously in the Int. J. Dev. Biol.}

Amphioxus regeneration: evolutionary and biomedical implications

Ildikó M.L. Somorjai

Int. J. Dev. Biol. (2017) 61: 689-696

https://doi.org/10.1387/ijdb.170219is

Regeneration in spiralians: evolutionary patterns and developmental processes

Alexandra E. Bely, Eduardo E. Zattara and James M. Sikes

Int. J. Dev. Biol. (2014) 58: 623-634

https://doi.org/10.1387/ijdb.140142ab

Segment formation in Annelids: patterns, processes and evolution

Guillaume Balavoine

Int. J. Dev. Biol. (2014) 58: 469-483

https://doi.org/10.1387/ijdb.140148gb

Hydra, a fruitful model system for 270 years

Brigitte Galliot

Int. J. Dev. Biol. (2012) 56: 411-423

https://doi.org/10.1387/ijdb.120086bg

Hydra, a model system to trace the emergence of boundaries in developing eumetazoans Angelika Böttger and Monika Hassel

Int. J. Dev. Biol. (2012) 56: 583-591

https://doi.org/10.1387/ijdb.113454ab

The head organizer in Hydra

Hans R. Bode

Int. J. Dev. Biol. (2012) 56: 473-478

https://doi.org/10.1387/ijdb.113448hb

Comprehensive gene expression analyses in pluripotent stem cells of a planarian, Dugesia japonica

Norito Shibata, Tetsutaro Hayashi, Ryutaro Fukumura, Junsuke Fujii, Tomomi KudomeTakamatsu, Osamu Nishimura, Syozo Sano, Fuyan Son, Nobuko Suzuki, Ryoko Araki, Masumi Abe and Kiyokazu Agata

Int. J. Dev. Biol. (2012) 56: 93-102

https://doi.org/10.1387/ijdb.113434ns

5 yr ISI Impact Factor $(2016)=2.421$
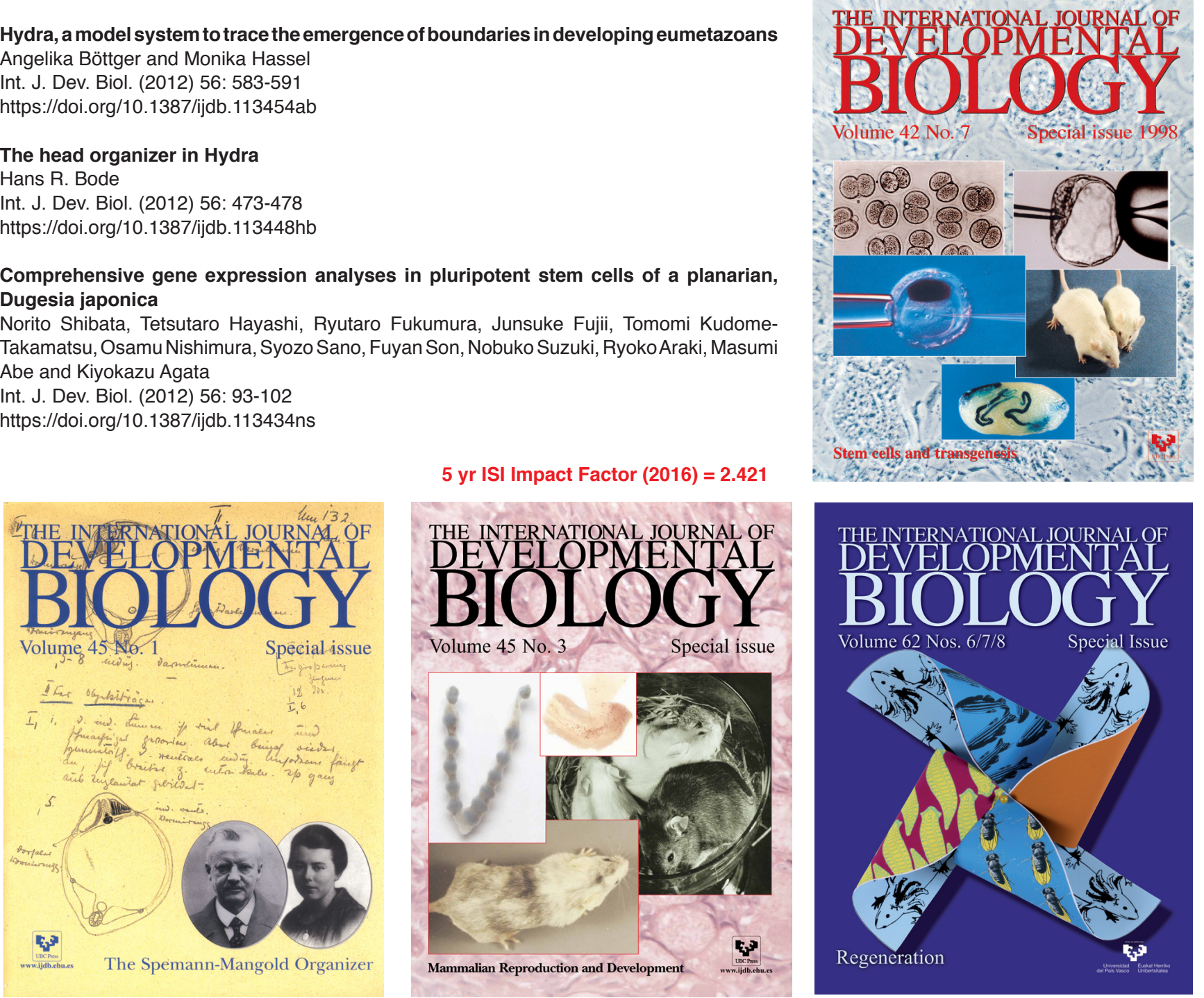